\title{
Maturity Models in the Age of Industry 4.0 - Do the Available Models Correspond to the Needs of Business Practice?
}

\author{
Vanessa Felch \\ University of Bamberg, Germany \\ vanessa.felch@uni-bamberg.de
}

\author{
Björn Asdecker \\ University of Bamberg, Germany \\ bjoern.asdecker@uni-bamberg.de
}

\author{
Eric Sucky \\ University of Bamberg, Germany \\ eric.sucky@uni-bamberg.de
}

\begin{abstract}
Maturity models (MMs) enable users to identify the need for change and to derive the necessary measures to accompany the change process. Existing literature reviews indicate that the number of available models has increased sharply in recent years. At the same time, it is found that the number of model applications does not keep up with the pace of development. Against the background of the current digitization trend, this article empirically investigates which models are actually used in business practice. We find that the degree of application is very low. Moreover, we also examine user-related model requirements, reasons for employing MMs, and the purpose of using MMs, which can support the user-centered development of future MMs.
\end{abstract}

\section{Introduction}

Since their first manifestations in the 1970s, MMs have developed into an important management tool with a wide range of applications [31]. The models provide a structured approach to initiate and accompany short-term operational projects, mediumterm tactical changes or long-term strategic change. All models are built on the hypothesis that organizational evolution follows a predictable linear stage-by-stage pattern [17]. Therefore, MMs describe "[...] an anticipated, desired, or typical evolution path of these objects shaped as discrete stages" ([4], p. 213). They imply a path to excellence and provide guidelines for the realignment, reconfiguration, and renewal of existing capabilities [28].

One current trend that will require many companies to evolve is digitization. In manufacturing, the Internet of Things (IoT) and cyber-physical systems (CPS) in particular are disruptively changing the industry ([42],[30],[33]). Both IoT and CPS in combination with other technologies such as big data and machine learning foster automation and digitization of production systems [13]. The German federal government called this new era the fourth industrial revolution, or simply "Industry 4.0 (I4.0)" ([15],[38]), which represents a subarea of digitization in manufacturing. While acknowledging that a commonly accepted definition does not exist, for the purpose of this paper, we refer to Zhou et al. and define I4.0 as the interplay of state-of-the-art technologies (e.g., IoT, CPS, big data) to increase the automation and digitization of the manufacturing processes, which facilitate a highly flexible production model of personalized and digital products and services, with real-time interactions between people, products and devices during the production process [44]. Once a German peculiarity, the concept of I4.0 has prevailed. Today, industry leaders such as General Electric, Cisco, Intel, and IBM have adopted the buzz word to effectively refer to the current digital industrial transformation in manufacturing [36].

To successfully master the upcoming changes, researchers and consulting firms have developed a variety of MMs in recent years. This paper aims to give an overview of available I4.0 MMs and to empirically test their dissemination in business practice. In particular, we aim to address the following two research questions:

RQ1: Which I4.0 MMs (scientific models and models of management consultancies) are currently available?

RQ2: How prevalent are these MMs in business practice and how are they perceived by users and nonusers?

This study contributes to the literature by not only complementing existing comprehensive literature reviews with an I4.0 perspective but also exploring the user needs with regard to MMs. Some scholars have presented general design principles for MMs [31]. While such frameworks are important to ensure quality during model development, it should be noted that they 
are "[...] based on existing literature [...]" ([31], p. 11) and do not include the user perspective. Since the consideration of user needs is crucial for the dissemination and success of a MM, it is very surprising that there have been only few empirical studies on this topic so far.

To the authors' best knowledge, the publication that is most similar to this one originated from Jamaluddin et al. [19]. They carried out an online survey with a total of 43 responses to investigate the level of project management MM awareness, its extent of adoption and the necessary prerequisite requirements for the use of MMs in the information and communication technology industry in Malaysia. Our study transfers the idea of this paper to the context of I4.0.

\section{Background}

\subsection{Maturity models}

Many companies are exposed to growing market dynamics and thus to increasingly intense competition. Therefore, organizations are under great pressure to evolve in line with the constantly changing market conditions. As pointed out in the introduction, MMs can contribute to organizational transformation and renewed competencies in organizations by initiating a change process [28]. In particular, MMs are adequate tools for (1) documenting the status quo, (2) developing a corporate vision for process excellence and providing guidance on that development path, and (3) comparing capabilities between business units and organizations [11]. It is thus not surprising that an increasing number of MMs has been developed and presented in the literature [40]. Pöppelbuß and Röglinger even raised the question "[...] whether high quantity goes along with high quality" ([31], p. 1). Against this background, three recent comprehensive reviews have tried to bring order to the available literature.

Pöppelbuß et al. reviewed 76 articles from leading information systems journals and conference proceedings with a specific focus on MMs that were published between 1996 and 2010 [32]. The authors investigated MMs from three perspectives: research, publication, and practice. They noted that the level of research and publication activity has generally been increasing. Moreover, they reported a lack of empirical validity and ready-to-use documentation. Academia often presents new MMs in a state that is not sufficient for direct application. More specifically, they fall short in providing detailed guidelines to support the model's application.
Wendler structured and analyzed the current MM research literature in a mapping study [40]. He identified 237 relevant articles that have been published between 1993 and 2010. The study showed that most publications refer to the development of MMs and empirical studies. Articles were considered empirical if they had been conducted to develop, apply or validate MMs. Wendler highlighted " $[\ldots]$ that larger (quantitative) studies about the applicability of maturity models are scarce" ([40], p. 1324). Moreover the author noted that the Capability Maturity Model Integration (CMMI) "[...] is obviously the only 'standard' maturity model really noticed within the academic community" ([40], p. 1328). Other models from standardization organizations, such as ISO 9000 or the Object Management Group's Business Process Maturity Model (BPMM), play a subordinate role and have been only rarely addressed in research. Moreover, he showed that MMs are used in more than 20 application domains with a strong focus on software development and software engineering, followed by project management application domains.

More recently, Tarhan et al. considered studies that were published between 1990 and 2014 in academic journals, conference proceedings, and books [37]. Based on their search of digital libraries, they initially retrieved 2,899 references, with 61 of those considered relevant for further analysis. In line with Wendler [40], they reported that most previous publications established a MM, showed the application of a model or compared different models. In addition, Tarhan et al. [37] called for more prescriptive rather than descriptive models. They argued that a major prerequisite for the fulfillment of its prescriptive purpose is extensive documentation that lays out specific process areas, goals, best practices, and achievement measures. Furthermore, these authors also emphasize that extensive models might deter decision makers because the models demand greater efforts for adoption.

While the reviews thus provide a preliminary overview of user requirements, none of the cited studies has analyzed this in a structured manner. This paper is intended to contribute to this research gap.

\subsection{Industry 4.0 maturity models}

Since the effects of digitization and particularly I4.0 have only been apparent in recent years, the previously introduced literature reviews could not take such MMs into account. Because I4.0 has a strong IT and software background and is often implemented as part of projects, we believe that I4.0 is an appropriate application domain for MMs. Therefore, with regard to RQ1, this paper aims to complement the previous reviews by focusing on such publications. The 
identified models are then empirically investigated for their dissemination in business practice.

Since digitization is highly practice-driven, there are not only scientific MMs but also approaches from practitioners and other stakeholders. Particularly worth mentioning are the models of management consultancies, which often act as intermediaries between theory and practice and accompany change processes as external, independent institutions. Typically, MMs are used as a diagnostic tool at the beginning of a consulting project or to certify certain capabilities [32].

To identify relevant scientific MMs, a literature search has been conducted. Subsequently, an overview of the most prominent I4.0 MMs from management consultancies is given.

Scientific MMs for Industry 4.0. The literature search has been conducted in five common digital libraries from December 2017 to January 2018: EBSCOhost, Emerald Insight, ScienceDirect, Wiley, and Google Scholar. The search terms included "Industry 4.0", "Industrial Internet", "I4.0”, "Internet

\section{Table 1. Overview of scientific Industry 4.0 MMs}

[7] DeCarolis et al. - The Digital Readiness Assessment Maturity Model (DREAMY): DREAMY pursues the digitization of manufacturing firms. The model architecture consists of five maturity levels (initial; managed; defined; integrated and interoperability; digital-oriented) and four dimensions (process; monitoring and controlling; technology; organization).

[12] Ganzarain and Errasti - Three Stages Maturity Model in SME's towards Industry 4.0: The model describes a strategic development path for the digitization of SME. The model is composed of five maturity stages (initial; managed; defined; transform; detailed business model) and three dimensions (envision; enable; enact).

[14] Gökalp et al. - Industry 4.0-Maturity Model: The model for manufacturing processes contains six stages (incomplete; performed; managed; established; predictable; optimizing) and five dimensions (asset management; data governance; application management; process transformation; organizational alignment).

[18] Jeeger and Halse - The IoT Technological Maturity Model: The model determines the current IoT implementation level for manufacturing enterprises. It introduces eight maturity stages (3.0 maturity; initial to 4.0 maturity; connected; enhanced; innovating; integrated; extensive; 4.0 maturity), which are applied to the adoption of IoT technology. Thus, the model has only one dimension.

[20] Katsma et al. - Supply Chain Systems Maturing towards the Internet of Things (IoT): The model focuses on the information and communication technology (ITC) deployment. It contains four stages that describe the development from Enterprise Resource Planning (ERP) to the IoT (ERP; ERP 2.0; SOA/SAAS; IoT). The stages are applied to four different dimensions (business; application; information; technical infrastructure).

[21] Klötzer and Pflaum - Maturity Model for Digitalization: The authors distinguish between two facilitators of digital transformation: smart product realization and smart product application, which leads to two MMs. Both consist of five stages (digitalization awareness; smart networked products; the service-oriented enterprise; thinking in service systems; the data-driven enterprise) and cover nine dimensions with only minor differences (strategy development; offering to the customer; "smart" product/factory; complementary IT system; cooperation; structural organization; process organization; competencies; innovation culture).

[23] Leyh et al. - System Integration Maturity Model Industry 4.0 (SIMMI 4.0): SIMMI 4.0 enables organizations to evaluate I4.0 IT capabilities. It consists of five stages (basic digitalization level; cross-departmental digitalization; horizontal and vertical digitalization; full digitalization; optimized full digitalization) with four dimensions (vertical integration; horizontal integration; digital product development; cross-sectional technology).

[35] Schumacher et al. - Industry 4.0 Maturity Model: The model is designed for manufacturing companies. It consists of five maturity stages. However, the authors describe only the first and the fifth level (level 1: complete lack of attributes; level 5: state-of-the-art of required attributes). The maturity stages are applied to nine dimensions (strategy; leadership; customers; products; operations; culture; people; governance; technology).

[39] Weber et al. - Maturity Model for Data-Driven Manufacturing (M2DDM): M2DDM analyzes the IT architecture of manufacturing companies to provide a development path towards servitization. They suggest five stages (non-existent IT integration; data and system integration; integration of cross-life-cycle data; service-orientation; digital twin up to a selfoptimizing factory). The model focuses entirely on IT systems, that is, it has only one dimension.

[41] Westermann et al. - Maturity Levels for Cyber-Physical Systems: The model targets organizations that try to build CPS capabilities. It consists of two layers. The first layer provides a general model with five stages (monitoring; communication and analysis; interpretation and services; adaption and optimization; cooperation) on a single overall CPS dimension. The second level is more detailed and provides 4-5 maturity stages for single CPS components, which act as dimensions (actuatory; sensory (signal characteristics, signal source); information processing; communication system (vertical communication, horizontal communication, connectivity, network connection); human-machineinterface (functionality, adaptability, location); data (data storage, location of storage, use of external data); services). 
of Things", "IoT", "Cyber-Physical Systems", and "CPS" combined with "maturity model" and "capability model". To ensure academic rigor, only peer-reviewed publications (journals and proceedings) in English language were selected. The abstracts of all identified references were analyzed with regard to their relevance. A publication was considered for further analysis if it presented a full model including maturity stages and dimensions, because without this information, it would not be applicable. Ultimately, ten studies were selected (see Table 1).

Consultancy MMs for Industry 4.0. Due to the enormous number of management consultancies, we only consider the MMs of the leading ones, since it can be assumed that their models are most widespread in practice. According to Consultancy.uk [6], the big ten (PricewaterhouseCoopers (PwC), Deloitte, Ernst \& Young (EY), KPMG, McKinsey \& Company, Boston Consulting Group (BCG), Accenture, IBM, Microsoft, and Booz Allen Hamilton) account for almost $56 \%$ of the industry's $\$ 150$ billion global market.

Initially, their homepages were browsed for the same terms that had been used for the literature search. However, we found that consultancies have only made more general MMs publicly available. Despite their broader focus we are confident that they could still be applied to the investigated manufacturing context. For this reason, the search terms were changed to "digitization" in combination with "maturity model" or "capability model", which led to a total of 10 generic

\section{Table 2. Overview of Industry 4.0 consultancy MMs}

[1] Accenture - Digital Capability Assessment (DCA): The tool analyzes digital capabilities needed to compete in today's and tomorrow's markets. It considers five dimensions (strategy and leadership; people and culture; product and service; customer experience; enterprise enablement). The model stages are not publicly available.

[5] BCG - Digital Acceleration Index (DAI): DAI identifies opportunities for accelerating the digital transformation. The model describes four stages (digital passive; digital literate; digital performer; digital leader). The four building blocks (business strategy driven by digital; digitize the core; new digital growth; enablers) with 37 subbuilding blocks (e.g., priorities \& alignment, digital supply chain, shared services) cover the entire value chain from strategy to capabilities.

[8] Deloitte - Digital Maturity Model (DMM): DMM represents the first cross-organizational digital MM. Five core business dimensions (customer; strategy; technology; operations; organization and culture) and 28 subdimensions (e.g., customer experience, security) are used to assess the digital capability. Model stages are not publicly available.

[10] EY - Digital Readiness Assessment: The model verifies the organization's strategy and provides an improvement plan towards a fully digital organization. The evaluation is based on seven focus areas (strategy, innovation and growth; customer experience; supply chain and operations; technology; risk and cyber security; finance, legal and tax; people and organization). The model contains three stages (developing; established; leading).

[29] IBM - Big data \& Analytics Maturity Model: The model supports organizations by evaluating their current capabilities to generate value from large data investments in support of strategic business initiatives. The model consists of five stages (ad hoc; foundational; competitive; differentiating; breakaway) and six dimensions (business strategy; information; analytics; culture \& execution; architecture; governance).

[16] IBM - Smart Grid Maturity Model (SGMM): SGMM comprises five stages (exploring and initiating; functional investing; integrating - cross functional; optimizing - enterprise wide; innovating - next wave of improvements), which are applied to eight domains (strategy, management and regulatory; organization; technology; societal and environment; grid operations; work and asset management; customer management and experience; value chain integration).

[22] KPMG - Digital Readiness Assessment (DRA): DRA assesses the organization's relevant sections from two different perspectives: transformation intensity and operational effectiveness. Both perspectives have four dimensions (development and purchasing; production; marketing; sales) with four stages (reactive participant; digital operator; ambitious transformer; smart digitalist).

[25] McKinsey \& Company - Digital Capabilities (DC): DC is a tool to benchmark the digital capabilities for facilitating a digital strategy. The capabilities are represented by six dimensions (data-driven insights; integrated customer experience; digital marketing; digitally-enabled operations; next-gen technology; digital enablers). Maturity stages are not publicly available.

[26] McKinsey \& Company - Digital Quotient (DQ): DQ analyzes digital strengths and weaknesses across business units and prioritizes those with the greatest influence. The performance is evaluated based on four dimensions (strategy; culture; organization; capabilities) with several subdimensions (e.g., risk appetite, connectivity). Maturity stages are not publicly available.

[34] PWC - Industry 4.0 / Digital Operations Self Assessment: The model focuses on the industrial capabilities across the organization. The assessment considers six dimensions (business models, product and service portfolio; market and customer access; value chains and processes; IT architecture; compliance, legal, risk, security and tax; organization and culture) with four stages (I-digital novice; II-vertical integrator; III-horizontal collaborator; IV-digital champion). 
models (see Table 2). It should be noted that most consultancies treat their MMs as intellectual property and do not make the complete version publicly accessible. We accepted this fact for consultancy MMs since these models will still be applied within the scope of the consulting services offered. The ten identified scientific and ten identified consultancy MMs are now empirically examined with regard to their dissemination in business practice. The methodology of the survey will be introduced in the next section.

\section{Methodology of the empirical study}

With regard to RQ2, we pursued a quantitative empirical research approach. A web-based questionnaire was used to collect data because of its dynamic routing possibilities. The questionnaire consisted of two parts. First, participants were asked a maximum of 13 questions about MMs in general and I4.0 MMs in particular (see Table 3), followed by demographic questions (e.g., gender, I4.0 experience). The models cited in Q3 cover most frequently investigated models in the literature, namely, CMMI, ISO 9000, and BPMM [40]. The models asked for in Q6 refer to the 20 models determined in the literature overview. The decision of whether a participant will be referred to question Q11 or Q12 depends on whether the application of at least one model was indicated in Q3 or Q6 (user $\rightarrow$ Q11, nonuser $\rightarrow$ Q12).

The provided answer options for Q11, Q12, and Q13 were based on available literature. For instance,
Pöppelbuß and Röglinger [31] noted that the purposes of MMs can be descriptive, prescriptive and/or comparative, which was queried in the context of Q11. Furthermore, Jamaluddin et al. [19] served as the basis for the answer options of Q12 and Q13, which were supplemented by the inclusion of the most frequent criticisms of MMs ([4],[2],[27]). The questionnaire was provided in English and German to allow for international participants. Before the field phase, the survey was pretested by five experts and minor changes were made with regard to wording and question sequence.

Two acquisition channels were used to address potential participants: (1) German industrial associations and (2) professional social networks. The industrial associations ZVEI (https://www.zvei.org/), BME (https://www.bme.de), and bdvb (https://www.bdvb.de/) distributed the link to the online questionnaire via their newsletter and social media accounts. Moreover, the link was spread in I4.0 groups on two of the most popular professional social networks (XING and LinkedIn).

The survey was open for participation from May to mid-June 2018. In total, 894 people clicked on the provided invitation URL, of whom 165 finished it, implying a completion rate of $18.5 \%$. The average response time was approximately 8 minutes. Most participants $(84.8 \%)$ work for large corporations with more than 250 employees and a turnover of $€ 50$ million. The majority indicated that they work for manufacturing firms $(49.1 \%)$, followed by industry associations $(26.1 \%)$, consulting firms $(16.4 \%)$, and

Table 3. Questionnaire and dynamic routing

\begin{tabular}{l|l|l}
\hline ID & $\begin{array}{l}\text { Question (to prevent random responses and an increase in drop-outs, } \\
\text { participants were not forced to provide answers) }\end{array}$ & Routing \\
\hline Q1 & $\begin{array}{l}\text { Which of the following capabilities do you think are necessary for your } \\
\text { organization to remain competitive in the digital future? }\end{array}$ & $\rightarrow$ Q2 \\
\hline Q2 & Did you get in touch (heard of / applied) with MMs in general? & if yes $\rightarrow$ Q3, else $\rightarrow$ end \\
\hline Q3 & $\begin{array}{l}\text { Which of the following prominent IT and/or process-related MMs do you } \\
\text { know and to what extent? }\end{array}$ & if 1+ model applied $\rightarrow$ Q4, else $\rightarrow$ Q5 \\
\hline Q4 & How likely is it that you would recommend the [model] to your colleague? & $\rightarrow$ Q5 \\
\hline Q5 & Did you get in touch (heard of / applied) with Industry 4.0 MMs? & if yes $\rightarrow$ Q6, else $\rightarrow$ Q11/Q12 \\
\hline Q6 & Which of the following Industry 4.0 MMs do you know and to what extent? & if 1+ model applied $\rightarrow$ Q7, else $\rightarrow$ Q8 \\
\hline Q7 & How likely is it that you would recommend the [model] to a colleague? & $\rightarrow$ Q8 \\
\hline Q8 & Does your organization use a self-developed Industry 4.0 MM? & if yes $\rightarrow$ Q9, else $\rightarrow$ Q11/Q12 \\
\hline Q9 & Has your organization developed the Industry 4.0 MM itself? & $\rightarrow$ Q10 \\
\hline Q10 & What were the reasons for an organization-specific solution? & $\rightarrow$ Q11 \\
\hline Q11 & What is your purpose of using maturity models? & $\rightarrow$ Q13 \\
\hline Q12 & What are the reasons for not applying MMs? & $\rightarrow$ Q13 \\
\hline Q13 & What requirements do you have concerning MMs? & $\rightarrow$ end \\
\hline
\end{tabular}


academia $(4.2 \%)$. The organizations' headquarters are located almost exclusively in Europe $(93.3 \%)$. Since top-level jobs are primarily held by men [9], the participants were also predominantly male $(86.1 \%)$. Despite the novelty of the concept, over half of the respondents $(60.0 \%)$ indicated that they have been working on I4.0-related projects for more than two years. We are therefore confident that the sample reflects the necessary expertise to obtain valid inferences on the degree of awareness of the I4.0 models.

Before further analyses, the data were examined for potential late-response and nonresponse biases [3]. Concerning late-response, the answers of the early participants were compared with those of the late participants. To test for nonresponse bias, we compared the responses of dropouts with those who completed the survey. All tests were statistically insignificant on a $\alpha=0.05$ level. Therefore, we are confident that neither late-response nor nonresponse plays a role in this study.

\section{Results and discussion}

At the beginning (Q1), participants were asked to assess the relevance of necessary competencies to remain competitive in the digital future on a five-point Likert scale (+1 "not important" to +5 "very important"). Accordingly to their responses, it is most important to determine the current digitization capabilities $(Q 4.21, \mathrm{SD}=0.83, \mathrm{n}=163)$, followed by the ability to establish an improvement path towards digitization excellence $(\propto 4.07, \mathrm{SD}=0.95, \mathrm{n}=165)$, to benchmark digitization capabilities within one's organization $(\otimes 3.73, \mathrm{SD}=0.99, \mathrm{n}=164)$, and to perform external benchmarking between companies and industries $(\propto 3.60, \mathrm{SD}=1.13, \mathrm{n}=165)$. In principle, these capabilities correspond to the purpose of MMs [31]. The answers are therefore a strong indicator of the need for suitable I4.0 models. Although many decision-makers would need a MM based on these answers, its use in practice is unexpectedly low.

Only approximately one-third (38.2\%) of respondents had heard of or even used MMs, while almost two-thirds $(61.8 \%)$ of respondents had not gotten in touch with any MM (Q2). Before turning to specific I4.0 MMs, this study (Q3) empirically examines the utilization of and satisfaction with the MMs that are considered the most prominent according to the literature [40]. Among these, ISO 9000 has the highest degree of dissemination, followed by BPMM and CMMI (see Figure 1). This result is surprising because most literature concentrates on CMMI [40].
Since quantity says nothing about quality, we believe that it is necessary to suggest a performance indicator with respect to user satisfaction. In this regard, we referred to the well-established net promoter score (NPS) and adapted it to MMs (see Q4). A model's NPS is calculated by subtracting the percentage of detractors (0-6) from the percentage of promoters (9-10). However, a certain number of users is necessary to be able to make meaningful statements, which is only the case with ISO 9000 . For this model, the NPS is $-36.8 \%(n=20)$, which indicates that most users appear unsatisfied with the model and its results.

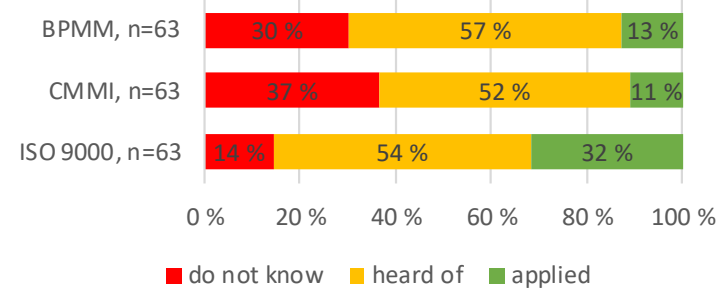

\section{Figure 1. Awareness level of prominent MMs}

Almost three-quarters (74.6\%) of respondents who know about the three most-common MMs have also heard of or applied I4.0 MMs (Q5). Obviously, there is currently no dominant scientific or consultancy model (Q6, see Figure 2 and Figure 3). Particularly striking is the small number of applications. In our sample, only five scientific models ([14],[21],[23],[35],[41]) have been applied in practice. The picture is only slightly better for the models of the consulting firms. Among the three best-known MMs are McKinsey's Digital Capabilities MM [25], IBM's Big data \& Analytics MM [29], and PWC's I4.0/Digital Operations Self Assessment [34]. Unfortunately, the application of the NPS failed due to the small number of actual users (Q7). One reason for the low prevalence in practice could be that the available models do not meet the needs of users, which leads to the development of organization-specific models (Q8-Q10).

In fact, more than half $(52.1 \%, \mathrm{n}=24)$ of the respondents who have gotten in touch with I4.0 MMs use such organization-specific models. The majority ( $66.7 \%, n=16)$ developed the model themselves; onethird $(33.3 \%, \mathrm{n}=8)$ used external help (e.g., consulting firms). The reasons for the development varied. On a five-point Likert scale $(+1$ "not important" to +5 "very important") the adjustability of such MMs to the organization's particularities was considered most important $(\propto 4.35, \mathrm{SD}=0.83, \mathrm{n}=23)$. Slightly less important were the independence of third parties ( $3.35, \mathrm{SD}=1.37, \mathrm{n}=23$ ), cost-benefit considerations (Q 3.26, $\mathrm{SD}=1.36, \mathrm{n}=23$ ), and potential competitive advantages, because such models cannot be replicated 


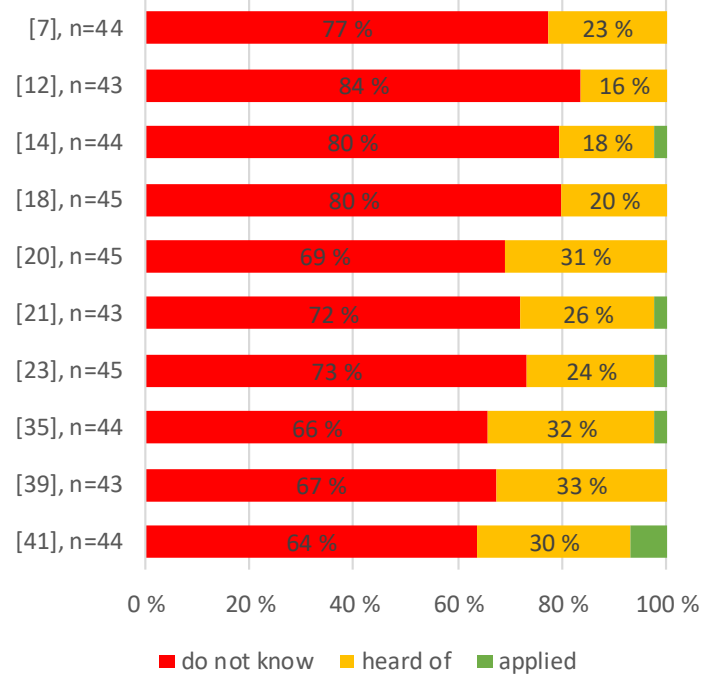

Figure 2. Awareness level of the Industry 4.0 scientific MMs

by competitors $(\propto 3.14, \mathrm{SD}=1.17, \mathrm{n}=22)$. Interestingly, the least relevant reason was the unavailability of suitable models $(\propto 2.64, \mathrm{SD}=1.14, \mathrm{n}=22)$.

In the following, participants who have applied at least one of the general or I4.0 MMs were asked (Q11) for the purpose of application (multiple answers possible, $\mathrm{n}=32$ ). Most respondents indicated a descriptive $(71.9 \%)$ and prescriptive $(75.0 \%)$ purpose. That is, they use MMs to determine the status quo and to derive a development path towards excellence. Despite frequent mention in the literature, comparative purposes play a subordinate role. Specifically, only one quarter uses MMs for internal $(25.0 \%)$ or external $(21.9 \%)$ benchmarking. Therefore, this study supports Tarhan et al.'s call for more prescriptive models [37].

In contrast, respondents who have not yet applied

\section{Table 4. Reasons for not applying MMs}

\begin{tabular}{l|c}
\hline Reason & Avg., SD, $\mathbf{n}$ \\
\hline Cost factor (acquisition) & $-0.23,0.97, \mathrm{n}=30$ \\
\hline Cost factor (application) & $+0.20,1.06, \mathrm{n}=30$ \\
\hline Do not understand the model & $-0.10,0.88, \mathrm{n}=30$ \\
\hline Limited resources & $-0.07,1.17, \mathrm{n}=30$ \\
\hline Limited time & $+0.10,1.03, \mathrm{n}=30$ \\
\hline MMs not useful & $-0.68,1.01, \mathrm{n}=31$ \\
\hline MMs not required at the moment & $-0.80,1.03, \mathrm{n}=30$ \\
\hline Too standardized, not customizable & $+0.31,0.97, \mathrm{n}=29$ \\
\hline Too strategic, no operational focus & $+0.43,1.01, \mathrm{n}=30$ \\
\hline
\end{tabular}

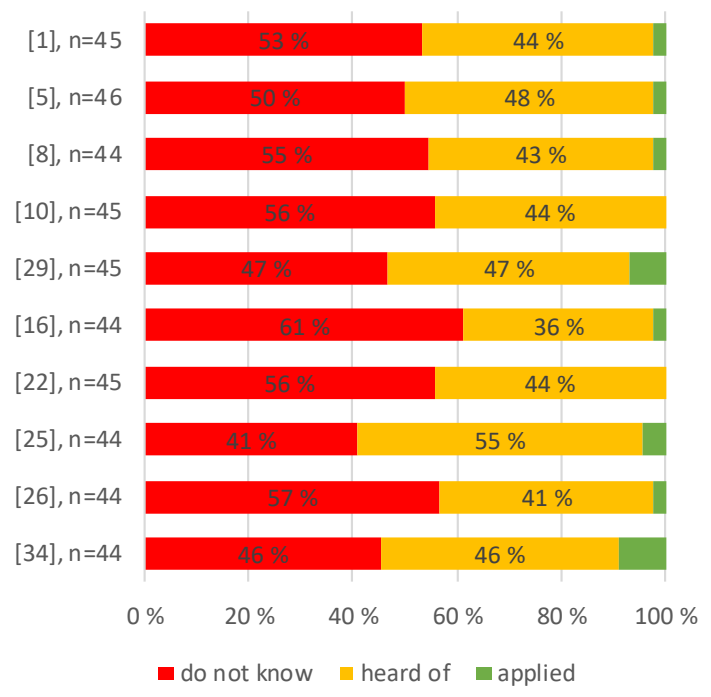

Figure 3. Awareness level of the Industry 4.0 consultancy MMs

any $\mathrm{MM}$ were asked (Q12) to indicate their reasons on a five-point Likert scale (-2 "strongly disagree" to +2 "strongly agree"). The results (see Table 4) show that current MMs are considered too strategic $(Q+0.43$, $\mathrm{SD}=1.01, \mathrm{n}=30)$ and lack customizability $(Q+0.31$, $\mathrm{SD}=0.97, \mathrm{n}=29)$. The least important reason is the lack of need for such models $(Q-0.80, \mathrm{SD}=1.03, \mathrm{n}=30)$ and their expected usefulness $(Q-0.68, \mathrm{SD}=1.01, \mathrm{n}=31)$.

In the last question of the survey's first part (Q13), MM users and nonusers were brought together to rate the importance of 14 model requirements on a fivepoint Likert scale $(+1$ "not important" to +5 "very important"). In general, process-orientation ( $Q 4.19$, $\mathrm{SD}=0.90, \mathrm{n}=62)$, quick application $(Q 4.11, \mathrm{SD}=0.83$, $\mathrm{n}=62)$, simplicity of adoption $(\otimes 4.10, \mathrm{SD}=0.92, \mathrm{n}=62)$, customizability $(\otimes 4.06, \quad \mathrm{SD}=0.89, \quad \mathrm{n}=62), \quad$ and providing a structured improvement plan ( $Q 4.05$, $\mathrm{SD}=0.86, \mathrm{n}=62$ ) are the five most important $\mathrm{MM}$ requirements (see Table 5). These results correspond to the findings of Jamaluddin et al. [19].

We also examined differences between MM users and nonusers with significant differences $(\alpha=0.05)$ with regard to the provision of a long-term improvement plan. Additionally, on a $\alpha=0.1-$ level, the requirements of providing specific improvement measurements, as well as the model's customizability, proved to be significantly more important to users than to nonusers. When compared with the available literature, it is particularly striking that the frequently demanded model validation, evaluation and provision of case studies appear to be of secondary importance from the practitioner's point of view ([40],[32],[37]). 
Table 5. Model requirements

\begin{tabular}{l|c|c|c}
\hline Model requirements (Avg, SD, $\mathrm{n})$ & $\begin{array}{c}\text { MM users } \\
(\text { Avg., } \mathrm{SD}, \mathrm{n})\end{array}$ & $\begin{array}{c}\text { MM nonusers } \\
(\text { Avg., } \mathrm{SD}, \mathrm{n})\end{array}$ & $\begin{array}{c}\text { t-test results } \\
\mathrm{t}(\mathrm{df})=\mathrm{t} \text { ratio, Sig. }\end{array}$ \\
\hline Available case studies $(3.37,1.12, \mathrm{n}=62)$ & $3.55,1.12, \mathrm{n}=31$ & $3.19,1.11, \mathrm{n}=31$ & $\mathrm{t}(60)=1.25, \mathrm{p}=0.22$ \\
\hline Aligned to business strategies $(3.92,0.93, \mathrm{n}=62)$ & $3.97,0.93, \mathrm{n}=32$ & $3.87,0.93, \mathrm{n}=30$ & $\mathrm{t}(60)=0.43, \mathrm{p}=0.67$ \\
\hline Aligned to industry standards $(3.87,1.00, \mathrm{n}=62)$ & $3.94,1.11, \mathrm{n}=32$ & $3.80,0.89, \mathrm{n}=30$ & $\mathrm{t}(60)=0.54, \mathrm{p}=0.59$ \\
\hline Customizability** $(4.06,0.89, \mathrm{n}=62)$ & $4.25,0.76, \mathrm{n}=32$ & $3.87,0.97, \mathrm{n}=30$ & $\mathrm{t}(60)=1.73, \mathrm{p}=0.08$ \\
\hline Generic use of terminology $(3.56,0.96, \mathrm{n}=61)$ & $3.68,1.11, \mathrm{n}=31$ & $3.50,0.78, \mathrm{n}=30$ & $\mathrm{t}(59)=0.72, \mathrm{p}=0.47$ \\
\hline Good documentation $(3.98,0.86, \mathrm{n}=62)$ & $3.97,0.93, \mathrm{n}=32$ & $4.00,0.79, \mathrm{n}=30$ & $\mathrm{t}(60)=-0.14, \mathrm{p}=0.89$ \\
\hline Process-orientation $(4.19,0.90, \mathrm{n}=62)$ & $4.22,0.98, \mathrm{n}=32$ & $4.17,0.83, \mathrm{n}=30$ & $\mathrm{t}(60)=0.23, \mathrm{p}=0.82$ \\
\hline Providing a structured improvement plan* $(4.05,0.86, \mathrm{n}=62)$ & $4.28,0.81, \mathrm{n}=32$ & $3.80,0.85, \mathrm{n}=30$ & $\mathrm{t}(60)=2.28, \mathrm{p}=0.03$ \\
\hline Providing improvement measurement** $(3.94,0.90, \mathrm{n}=62)$ & $4.13,0.79, \mathrm{n}=32$ & $3.73,0.98, \mathrm{n}=30$ & $\mathrm{t}(60)=1.74, \mathrm{p}=0.09$ \\
\hline Quick application / implementation $(4.11,0.83, \mathrm{n}=62)$ & $4.03,0.74, \mathrm{n}=32$ & $4.20,0.93, \mathrm{n}=30$ & $\mathrm{t}(60)=-0.80, \mathrm{p}=0.43$ \\
\hline Regular updates of maturity model $(3.56,0.93, \mathrm{n}=62)$ & $3.41,1.04, \mathrm{n}=32$ & $3.73,0.79, \mathrm{n}=30$ & $\mathrm{t}(60)=-1.39, \mathrm{p}=0.17$ \\
\hline Simplicity to adopt and administer $(4.10,0.92, \mathrm{n}=62)$ & $3.94,1.01, \mathrm{n}=32$ & $4.27,0.79, \mathrm{n}=30$ & $\mathrm{t}(60)=-1.42, \mathrm{p}=0.16$ \\
\hline Supply of assessment tool(s) $(3.80,0.89, \mathrm{n}=61)$ & $3.84,0.90, \mathrm{n}=31$ & $3.77,0.90, \mathrm{n}=30$ & $\mathrm{t}(59)=0.31, \mathrm{p}=0.76$ \\
\hline Validated maturity model and assessment $(3.71,0.98, \mathrm{n}=62)$ & $3.84,1.02, \mathrm{n}=32$ & $3.57,0.94, \mathrm{n}=30$ & $\mathrm{t}(60)=1.12, \mathrm{p}=0.27$ \\
\hline & \multicolumn{2}{|c|}{ Legend: $*=\mathrm{significant}$ on 0.05 -level; $* *=\operatorname{significant}$ on $0.1-\mathrm{level}$} \\
\hline
\end{tabular}

\section{Implications and outlook}

In his often-cited paper, Whetten suggested that the theoretical contribution of a paper could arise from the four core building blocks of theory development [43]: (1) the factors (variables, constructs, concepts) to explain the phenomenon of interest (what?), (2) the relationship between those factors (how?), (3) the logical justifications for altered views (why?), and (4) potential temporal and contextual features that limit generalizability (who, where, when?) [43]. Although there is no general theory about MMs or their application, this paper can be considered a starting point for such a study.

This paper sheds light on two previously overlooked topics: (1) the dissemination of MMs in business practice, and (2) (potential) user requirements. We found that particularly scientific I4.0 models are relatively unknown in the corporate world. This result is an indication that the large number of models published actually does not meet the user requirements and that the question "[...] whether high quantity goes along with high quality" asked by Pöppelbuß and Röglinger ([31], p. 1) probably is to be denied. It at least points to a communication problem, which should motivate researchers who are working on the development of MMs to progressively highlight the benefits for potential users.

While this paper does not provide a self-contained theory on the application of MMs, the data on the reasons for and against their application contribute to two of the four building blocks presented by Whetten by empirically testing the relevance of model requirements (what?) and reasons for not applying MMs (why?) [43]. These provisional findings can be used to specify causal relationships that help explain the application of MMs. Such theory appears important to constitute a new direction for model development. So far, the available literature only provides theorydriven design principles [31]. While such design principles are important to ensure academic rigor, user requirements should not be ignored. We therefore believe that our research can serve as an initial impetus for the development of user-centered design principles, which could then be used to integrate those two perspectives in future studies.

Furthermore, there are managerial implications. This study is a good starting point for practitioners, who are currently thinking about applying a MM to ensure the competitiveness of their company in the digital age ahead. We provide an overview of available I4.0 MMs, which complements existing comprehensive literature reviews. One of the greatest challenges decision makers face is to select the appropriate MM from the many available ones. To simplify this decision in the future, we suggested the adaption of the NPS concept as an appropriate key performance indicator for MM satisfaction. Although the number of survey participants was too small to make valid statements about the available I4.0 MMs, we are confident that practitioners can make use of the adapted NPS in their own evaluation efforts before choosing and applying a MM. In addition, all those 
who are disappointed with the existing models should take our study results as an opportunity to reflect on the development of their own MM. Unlike their standardized, generic counterparts, such idiosyncratic models cannot become common knowledge and are therefore more appropriate to generate sustained dynamic capabilities

Referring again to Whetten, future studies should focus on further exploring the relationship between identified factors (how?) and on possible temporal and contextual factors (who, where, when?) to achieve the overall goal of a MM theory [43]. Such research should also be able to overcome the limitations of this study, e.g., generalizability due to sample characteristics. For this reason, we are currently planning to conduct explorative qualitative research to learn about the causal relationships associated with the application of MMs and to replicate this study in other regions (e.g., North America or Asia).

\section{References}

[1] Accenture, "Digital Industry 4.0 Diagnostic", 2018, available from: https://www.accenture.com/il-en/insight -industrial-internet-things, accessed 09 June 2018.

[2] Albliwi, S.A., Antony, J. and Arshed, N. (2014), "Critical Literature Review on Maturity Models for Business Process Excellence", in International Conference on Industrial Engineering and Engineering Management, pp. 79-83.

[3] Armstrong, J.S. and Overton, T.S. (1977), "Estimating Nonresponse Bias in Mail Surveys", Journal of Marketing Research, Vol. 14 No. 3, pp. 396-402.

[4] Becker, J., Knackstedt, R. and Pöppelbuß, J. (2009), "Developing Maturity Models for IT Management - A Procedure Model and its Application”, Business and Information Systems Engineering, Vol. 51 No. 3, pp. 213-222.

[5] Boston Consulting Group, "Digital Acceleration Index", 2018, available from: https://www.bcg.com/ capabilities/technology-digital/digital-accelerationindex.aspx, accessed 09 June 2018.

[6] Consultancy.uk, "The 10 largest consulting firms in the world", 2017, available from: https://www.consultancy. uk/news/14018/the-10-largest-consulting-firms-in-theworld, accessed 09 June 2018.

[7] De Carolis, A., Macchi, M., Negri, E. and Terzi S. (2017), "A Maturity Model for Assessing the Digital Readiness of Manufacturing Companies", in Lödding, H., Riedel, R., Thoben, K.D., von Cieminski, G. and Kiritsis, D. (Ed.), "Advances in Production Management Systems. The Path to Intelligent, Collaborative and Sustainable Manufacturing. APMS 2017. IFIP Advances in Information and Communication Technology", Springer, Cham, pp. 1320.

[8] Deloitte, "Digital Maturity Model", 2018, available from: https://www2.deloitte.com/content/dam/Deloitte/
global/Documents/Technology-Media-

Telecommunications/deloitte-digital-maturitymodel.pdf, accessed 09 June 2018.

[9] Dezso, C., Ross, D. G. and Uribe, J. (2013), "Why are there so few women top managers? A large-sample empirical study of the antecedents of female participation in top management", working paper, Columbia Business School, Columbia University, New York, March 2013.

[10] Ernst \& Young, "Digital Readiness Assessment - Does your business strategy work in a digital world?", 2018, available from: https://digitalreadiness.ey.com/, accessed 09 June 2018.

[11] Felch, V., Asdecker, B. and Sucky, E. (2018), "Digitization in outbound logistics - Application of an Industry 4.0 Maturity Model for the Delivery Process", in 2018 30th Annual NOFOMA Conference, pp. 113128.

[12] Ganzarain, J. and Errasti, N. (2016), "Three stage maturity model in SME's toward Industry 4.0", Journal of Industrial Engineering and Management, Vol. 9 No. 5, pp. 1119-1128.

[13] Gilchrist, A. (2016), Industry 4.0: The Industrial Internet of Things, Apress, New York, NY.

[14] Gökalp, E., Şener, U. and Eren, P.E. (2017), "Development of an Assessment Model for Industry 4.0: Industry 4.0-MM", in Mas, A., Mesquida, A., O'Connor, R., Rout, T. and Dorling, A. (Ed.), "Software Process Improvement and Capability Determination. SPICE 2017. Communications in Computer and Information Science", Vol. 770, Springer, Cham, pp. 128-142.

[15] Hermann, M., Penetek, T. and Otto, B. (2016), "Design Principles for Industrie 4.0 Scenarios", in 2016 49th Hawaii International Conference on System Sciences, pp. 3928-3937.

[16] IBM, "Smart Grid Maturity Model: Creating a Clear Path to the Smart Grid", 2009, available from: $\mathrm{ftp}: / /$ public.dhe.ibm.com/software/in/industry/Smart_Gr id_Maturity_Model.pdf, accessed 09 June 2018.

[17] Isoherranen, V., Karkkainen, M.K. and Kess, P. (2015), "Operational Excellence Driven by Process Maturity Reviews: A Case Study of the ABB Corporation", in International Conference on Industrial Engineering and Engineering Management, pp. 1372-1376.

[18] Jæger, B. and Halse, L.L. (2017), "The IoT Technological Maturity Assessment Scorecard: A Case Study of Norwegian Manufacturing Companies", in Lödding, H., Riedel, R., Thoben, K.D., von Cieminski, G. and Kiritsis, D. (Ed.), "Advances in Production Management Systems. The Path to Intelligent, Collaborative and Sustainable Manufacturing. APMS 2017. IFIP Advances in Information and Communication Technology", Springer, Cham, pp. $143-150$.

[19] Jamaluddin, R., Chin, C.M.M. and Lee, C.W. (2010), "Understanding the Requirements for Project management Maturity Models: Awareness of the ICT Industry in Malaysia", in IEEE International Conference on Industrial Engineering and Engineering Management, pp. 1573-1577. 
[20] Katsma, C., Moonen, H. and van Hillegersberg, J. (2011), "Supply chain systems maturing towards the Internet-of-Things: a framework", in 24th Bled eConference eFuture: Creating Solutions for the Individual, Organisations and Society Proceedings, pp. 478-494.

[21] Klötzer, C. and Pflaum, A. (2017), "Toward the development of a maturity model for digitalization within the manufacturing industry's supply chain", in 2017 50th Hawaii International Conference on System Sciences, pp. 4210-4219.

[22] KPMG, "Digital Readiness Assessment", 2016, available from: https://assets.kpmg.com/content/dam/ $\mathrm{kpmg} / \mathrm{pdf} / 2016 / 04 / \mathrm{ch}$-digital-readiness-assessmenten.pdf, accessed 09 June 2018.

[23] Leyh, C., Schäffer, T., Bley, K. and Forstenhäusler, S. (2016), "SIMMI 4.0 - A Maturity Model for Classifying the Enterprise-wide IT and Software Landscape Focusing on Industry 4.0", in Federated Conference on Computer Science and Information Systems, pp. 1297-1302.

[24] McCormack, K., Ladeira, M.B. and Valadares de Oliveira, M.P. (2008), "Supply chain maturity and performance in Brazil", Supply Chain Management: An International Journal, Vol. 13 No. 4, pp. 272-282.

[25] McKinsey \& Company, "Strategy - Building a winning digital strategy", 2018, available from: https://www.mckinsey.com/solutions/digital-20-20/ourassessments/strategy, accessed 09 June 2018.

[26] McKinsey \& Company, "Strategy - Building a winning digital strategy", 2018, available from: https://www.mckinsey.com/solutions/digital-20-20/ourassessments/strategy, accessed 09 June 2018.

[27] Mettler, T. and Rohner, P. (2009), "Situational maturity models as instrumental artifacts for organizational design", in International Conference on Design Science Research in Information Systems and Technology.

[28] Mettler, T., Rohner, P. and Winter, R. (2010), "Towards a Classification of Maturity Models in Information Systems", in: D'Atri, A., De Marco, M., Braccini, A. and Cabiduu, F. (Ed.) "Management of the Interconnected World", Physica-Verlag, Heidelberg, pp. 333-340.

[29] Nott, C., "Big Data and Analytics Maturity Model", IBM, 2018, available from: http://www.ibmbigdatahub. com/blog/big-data-analytics-maturity-model, accessed 09 June 2018.

[30] Oesterreich, T. D. and Teuteberg, F. (2016), "Understanding the implications of digitization and automation in the context of Industry 4.0: A triangulation approach and elements of a research agenda for the construction industry", Computers in Industry, Vol. 83, pp. 121-139.

[31] Pöppelbuß, J. and Röglinger, M. (2011), "What makes a useful maturity model? A framework for general design principles for maturity models and its demonstration in business process management", in European Conference on Information Systems.

[32] Pöppelbuß, J., Niehaves, B., Simons, A. and Becker, J. (2011), "Maturity Models in Information Systems Research: Literature Search and Analysis",
Communications of the Association for Information Systems, Vol. 29, pp. 505-532.

[33] Posada, J., Toro, C., Barandiaran, I., Oyarzun, D., Stricker, D., de Amicis, R., Pinto, E. B., Eisert, P., Döllner, J. and Vallarino Jr., I. (2015), "Visual Computing as a Key Enabling Technology for Industrie 4.0 and Industrial Internet", IEEE Computer Graphics and Applications, Vol. 35 No. 2, pp. 26-40.

[34] PricewaterhouseCoopers, "Industry 4.0 - Enabling Digital Operations", 2018, available from: https://i4-0self-assessment.pwc.nl/i40/landing/, accessed 09 June 2018.

[35] Schumacher, A., Erol, S. and Sihn, W. (2016), “A Maturity Model for Assessing Industry 4.0 Readiness and Maturity of Manufacturing Enterprises", Procedia CIRP, Vol. 52, pp. 161-166.

[36] Stark, H., "Intel, IBM and Microsoft Get Candid About Industry 4.0", 2017, available from: https://www.forbes. com/sites/haroldstark/2017/03/09/intel-ibm-andmicrosoft-get-candid-about-industry-4-0/, accessed 23 August 2018.

[37] Tarhan, A., Turetken, O. and Reijers, H.A. (2016), "Business process maturity models: A systematic literature review", Information and Software Technology, Vol. 75, pp. 122-134.

[38] Wang S., Wan, J., Zhang, D., Li, D. and Zhang., C. (2016), "Towards smart factory for Industry 4.0: a selforganized multi-agent system with big data based feedback and coordination“, Computer Networks, Vol. 101, pp. 158-168.

[39] Weber, C., Königsberger, J., Kassner, L. and Mitschang, B. (2017), "M2DDM - A Maturity Model for DataDriven Manufacturing", Procedia CIRP, Vol. 63, pp. 173-178.

[40] Wendler, R. (2012), "The maturity of maturity model research: A systematic mapping study", Information and Software Technology, Vol. 54 No. 12, pp. 1317-1339.

[41] Westermann, T., Anacker, H., Dumitrescu, R. and Czaja, A. (2016), "Reference Architecture and Maturity Levels for Cyber-Physical Systems in the Mechanical Engineering Industry", in 2016 IEEE International Symposium on Systems Engineering, pp. 1-6.

[42] Weyer, S., Schmitt, M., Ohmer, M. and Gorecky, D. (2015), "Towards Industry 4.0 - Standardization as the crucial challenge for highly modular, multi-vendor production systems", IFAC-PapersOnLine, Vol. 48 No. 3," pp. 579-584.

[43] Whetten, D.A. (1989), "What Constitutes a Theoretical Contribution", Academy of Management Review, Vol. 14, No. 4, pp. 490-495.

[44] Zhou, K., Liu, T. and Zhou, L. (2015), "Industry 4.0 Towards future industrial opportunities and challenges", in 2015 12th International Conference on Fuzzy Systems and Knowledge Discovery, pp. 2147-2152. 